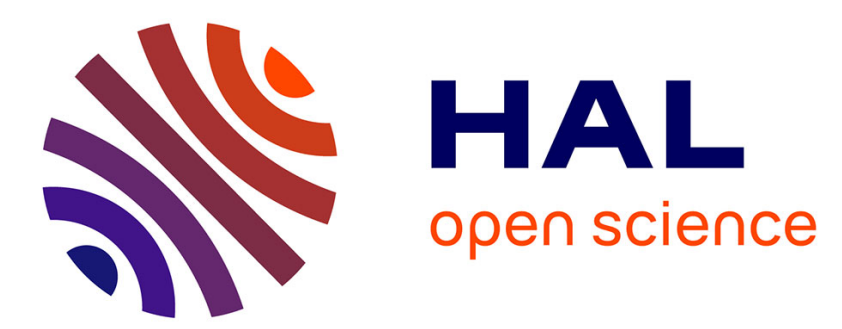

\title{
Deux inscriptions d'Aléria (Haute-Corse)
}

Paola Lombardi, Cinzia Vismara

\section{To cite this version:}

Paola Lombardi, Cinzia Vismara. Deux inscriptions d'Aléria (Haute-Corse). Gallia - Archéologie de la France antique, 2005, 62, pp.279-292. 10.3406/galia.2005.3071 . hal-01908355

\section{HAL Id: hal-01908355 https://hal.science/hal-01908355}

Submitted on 29 Jan 2020

HAL is a multi-disciplinary open access archive for the deposit and dissemination of scientific research documents, whether they are published or not. The documents may come from teaching and research institutions in France or abroad, or from public or private research centers.
L'archive ouverte pluridisciplinaire HAL, est destinée au dépôt et à la diffusion de documents scientifiques de niveau recherche, publiés ou non, émanant des établissements d'enseignement et de recherche français ou étrangers, des laboratoires publics ou privés.

\section{(ㅇ)(1) $\$$}

Distributed under a Creative Commons Attribution - NonCommercial - NoDerivatives| 4.0 


\title{
DEUX INSCRIPTIONS D'ALÉRIA (HAUTE-CORSE)
}

\author{
Paola LOMBARDI* et Cinzia VISMARA ${ }^{* *}$
}

Mots-clés. Corse, Aleria, inscription grecque, amende funéraire, flotte romaine, diplôme militaire.

Résumé. L'étude concerne deux inscriptions provenant d'Aleria et son territoire : un diplôme militaire et un texte funérciire en grec. Le diplôme, entier; mentionne une constitution déjà connue, celle du 18 féorier 129 ; les droits sont octroyés à un marin de la flotte de Misène, M. Cominius Cubesti f(ilius) Vielo, corse de Cobas (?). Le commentaire contient plusieurs observations sur la prosopographie des personnages mentionnés, ainsi que sur les militaires d'origine corse. Une annexe donne la liste des classiarii corses de la flotte de Misène, de la flotte de Ravenne el d'une flotte indéterminée; la liste d'autres classiarii altestés en Corse; un index des noms corses indigènes. L'inscription en grec (datable au plus tôt de la fin du IIr' s. apr. J.-C.) est un document privé, funéraire, dédié à une femme par son époux, tous deux grecs. Les éléments d'intérêt du document sont, d'une part, la présence en Corse, à une époque assez tardive, d'un personnage écrivant en grec et, d'autre part, la mention d'une amende funéraire à verser au hierotaton tamion en cas de violation du tombeau. Ces deux éléments trouvent une explication dans la présence à Aleria de marins de la flotte de Misène, d'origine gréco-orientale.

Key-words. Corsica, Aleria, Greek inscription, funerary fine, Roman fleet, military diploma.

Abstract. The article concerns two inscriptions from Aleria and its territory: a military diploma and a Greek funerary text. The diploma, entire, mentions a constitution already known, from 18 February 129. Rights are given to a seaman of Misene fleet, M. Cominius Cubesti $\mathrm{f}(\mathrm{ilius})$ Vielo a Corsican from Cobas (?). The commentary includes several observations on the prosopography of the individuals mentioned and on the soldiers from Corsica. An appendix gives the list of the classiarii of the Misenan and Ravenna fleets and of an indeterminate fleet, the list of other classiarii attested in Corsica, and an index of indigenous Corsican names. The inscription in (ireek (dated at least at the end of the $3^{\text {rd }}$ century $A D$ ) is a private funerary document for a Greek woman from her husband, himself Greek. The interesting part of this text is the presence in Corsica in a rather late period of somebody writing in Greek and the indication of a funerary fine to pay to the hierotaton tamion in case of a violation of the tomb. These two aspects can been explained by the presence in Aleria of seamen of the Misenan fleet of Creco-oriental origin.

Translation : Isabelle FAUI)ULT

Schlagwörter. Korsika, Aleria, griechische Inschrifl, Geldbuße im Friedhofsbereich, römische Flotte, Militärdiplom.

Zusammenfassung. Diese Studie beschäftigt sich mit zwei Inschriften, einem römischen Militärdiplom und einer griechischsprachigen Grabinschrift, die aus Alcria bzw. dem Umland der Stadt stammen. Das vollständig erhaltene Diplom erwähnt eine bereits bekannte Konstitution vom 18. Telruar des Jahres 129. In der Urkunde wird das Bürgerrecht verliehen an einen M. Cominius Cubesti $\mathrm{f}(\mathrm{ilius})$ Vielo, Korsen aus Cobas und einfachen Angehörigen der Flotte von Misenum. Unser Kommentar bietet verschiedene Beobachtungen zur Prosopographie der erwähnten Personen und zu römischen Militärangehörigen korsischer Herkunft. Im Anhang geben wir eine Liste der korsischen classiarii der Flotte von Misenum, der Flolte von Ravenna und einer unbestimmten Flolte, ferner eine Liste der übrigen auf Korsika bezeugten classiarii sowie ein Verzeichnis einheimischer korsischer Namen. Die nicht vor das späte dritte Jahrhundert n. Chr datierbare griechische Inschrift ist eine Privaturkunde aus dem Grabzusammenhang. Der Text bezieht sich auf eine Griechin und wurde von ihrem Gatten, ebenfalls Griechen, veranlasst. Zwei Aspekte sind an dem Dokument von besonderem Interesse, nämlich zum einen die Präsenz einer griechisch schreibenden Person auf Korsika während dieses relativ späten Zeitabschnitts, und zum anderen die Androhung einer an den hierotaton tamion zu entrichtenden Cieldbuße im Falle der Beschädigung der Crabslätle. Beides findet seine liklïrung in der Anwesenheit von Seeleuten griechisch-orientalischer Herkunft als Angehörige der Flotte von Misenum in Alcria.

Übersetzung : Stefan WIRTH

\footnotetext{
* Dipartimento di Scienze storiche, archeologiche e antropologiche dell'Antichità, Lniversità di Roma-La Sapienza, Piazzale Aldo Moro 5 , I-00185 Rome. Courricl : paola.lombardi@uniromal.it

** Dipartimento di Filologia e Storia, Università di Cassino, via Zamosch 43, I-03043 Cassino. Courriel : c.vismara@unicas.it
} 
Nous présentons ici deux inscriptions de la cité d'Aleria ${ }^{1}$ : un diplôme militaire provenant de son territoire et un texte grec qui fut trouvé à l'occasion de travaux archéologiques en marge du hameau actucl. Leur publication nous a été amicalement confiée par Joseph Cesari, conservateur régional de l'archéologie de la Corse, que nous remercions. Il s'agit d'une moisson particulièrement importante, pour une île si pauvre en témoignages épigraphiques.

\section{LE DIPLÔME MILITAIRE}

Le diplôme, conservé sur deux tablettes de bronze (fig. 1 et 2), fut trouvé dans le territoire d'Aléria (Haute-Corse) ct a été déposé auprès du Service régional de l'archéologie de Corse par le $\mathrm{D}^{\mathrm{r}}$ Jean-Félix Giudicelli.

\section{DESCRIPTION}

Dans la description dr. et g. se réfèrent à la face externe dans le sens de la lecture. Les surfaces sont recouvertes d'une patine verdâtre et présentent des irrégularités dues aux agents chimiques du terrain où les deux tablettes ont longtemps séjourné. Les deux plaquettes étaient encore superposées au moment où elles reçurent un coup (de pioche?) qui endommagea la tabella II : elle se trouvait donc à ce moment-là plus en surface, et en quelque sorte la tabella I fut protégée par l'autre.

La mise en page, ainsi que les lettres, sont bien soignées sur les surfaces principales, dont le champ est délimité par un cadre asse $z$ simple. On peut remarquer quelques I géants ; les lettres sont allongées, les traits internes des $\mathrm{M}$ se rejoignent à la base de la lettre. Des signes de ponctuation ne sont visibles que dans la formule onomastique du titulaire du document, sur la labella I, côté extérieur, et entre les praenomina et les gentilices des témoins, sur la tabella II, côté extérieur. Sur les surfaces non destinées à être vues, les lettres sont plus espacées et arrondies, et le ductus est moins soigné, mais nous ne pensons pas qu'elles ont été incisées par une autre main, comme c'est le cas dans d'autres diplômes (RMI), III, appendice 2, p. 339-340).

\section{TABELLA I}

H. $\max .: 19,1 \mathrm{~cm}$; larg. $\max .: 15,9 \mathrm{~cm}$; deux trous $(\varnothing$ : $0,5 \mathrm{~cm})$ à $9,5 \mathrm{~cm}$ de la marge supérieure, celui de g. à $5,3 \mathrm{~cm}$ de la marge g., l'autre à $4,8 \mathrm{~cm}$ de la marge dr.; poids $230 \mathrm{~g}$. Il manque l'angle inférieur dr.; on remarque des lacunes près des autres angles; les rebords sont rongés; il existe une cassure horizontale d'environ $4,5 \mathrm{~cm}$ vers le milicu du côté g. ; un coup a soulevé la surface un peu plus bas à g. du milicu de la tablette.

1. Le premier paragraphe est commun, le chapitre sur linscription en grec est dû à P. Lombardi, les autres ainsi que l'annexe à (: Vismara. Nous tenons à remercier les services techniques du Service régional de l'archéologie de Corse et, pour les conseils et les relectures, les professeurs : M. L. Caldelli, M. (. Granino, A. Mastino et 'T. Ritti. I.es abréviations des périodiques suivent celles de L'Année philologique.

\section{Extrinsecus (fig. 1a)}

Champ : $17 \mathrm{~cm} \times 13,9 \mathrm{~cm}$; larg. du cadre : $1,1 \mathrm{~cm} ; \mathrm{h}$. des lettres : $0,3-0,5 \mathrm{~cm}$; interligne : $0,2 \mathrm{~cm}(0,9 \mathrm{~cm}$ sur la ligne des trous entre les lignes 11 et 12).

I.e texte est encadré par un filet plat entre deux filets plus étroits.

[ $]$ mpp(erator) Caesar; diui Traiani Parthici f(ilius), diu[i]

Neruae nepos, Traianus Hadrianus A $u[g$ (ustus)], pontif(ex) max(imus), trib(unicia) potest(ate) $\overline{X I I}$, co(n)s(ul) $\overline{I I I}, p($ ater $) p($ atriae $)$

is (!) qui militauerunt in classe praeto $=$

5 ria Misenensi, quace est sub Iulio

Frontone, sex et uiginti stipendis eme =

ritis, dimissis honesta missione, quo $=$

rum nomina subscripta sunt, ipsis $l i=$

beris posterisque eorum ciuitatem

10 dedit et conubium cum uxoribus quas

tunc habuissent $[c]$ um est ciuitas iis

data, $[a]$ ut, si qui caelibes essent, cum

iis quas postea duxissent $[d]$ umtaxat

$\sin [g]$ ulis singulas. $A(n t e) d($ iem $) \quad \overline{X I I} k$ (alendas) Mart(ias)

15 I. [I] uuen[tio] Celso II Q. Iulio Balbo co(n)s(ulibus).

$$
\text { [l] } x \text { gregale }
$$

M. Cominio (ubestif. Vieloni corso cobas (io $\vdots$ )

et Taiae fill(iae) eius

et Tatiae fil (iae) eius.

20) Descriptum et recognitum ex tabula $a[e]=$

nea quae fixa est Romae in muro pos $[t]$

lemplum diui Aug(usti) ad Mineruam.

Intus (fig. 1b)

H. des lettres : $0,3-0,5 \mathrm{~cm}$; interligne : $0,2 \mathrm{~cm}(0,8 \mathrm{~cm}$ sur la ligne des trous).
[Im] p(erator) Caesar; diui Traian(i) Parthic(i) f(ilius).
diui Neru[ ae $]$
nepos, Traianus Hadrian(us) Aug(ustus), pontif(ex)
$\max$ (imus),
trib(unicia) potes(tate) $\overline{X I I}, \operatorname{co}(n) s(u l) I I I, p($ ater $)$
$p$ (atrice),
iis qui militauer (unt) in classe praetor(ia) Mis(enensi),
5 quae est sub Iulio Irontone, sex et ui =
ginti stip(endis) emer(itis), dimiss(is) honest(a) missione,
quori um) nomin(a) subscript(a) sunt, ipsis liber(is)
poster(isque) eor(um) ciuit(atem) ded(it) el comub(ium) cum uxor(ibus)
quas tunc hab(uissent) cum est ciuit (as) iis data
10) siqui caelib(es) ess(ent) cum iis quas post(ea) dux(issent)
$\bullet$
dumiaxat $\operatorname{sing}(u$ lis) singul(as).

\section{TABELLA II}

H. max.: $16 \mathrm{~cm}$; larg. max.: $18,9 \mathrm{~cm}$; deux trous ( $\varnothing: 0,5 \mathrm{~cm}$ environ) à $5,2 \mathrm{~cm}$ de la marge supéricure, celui de g. à $9,5 \mathrm{~cm}$ de la marge g., l'autre à $9,6 \mathrm{~cm}$ de la marge dr. 

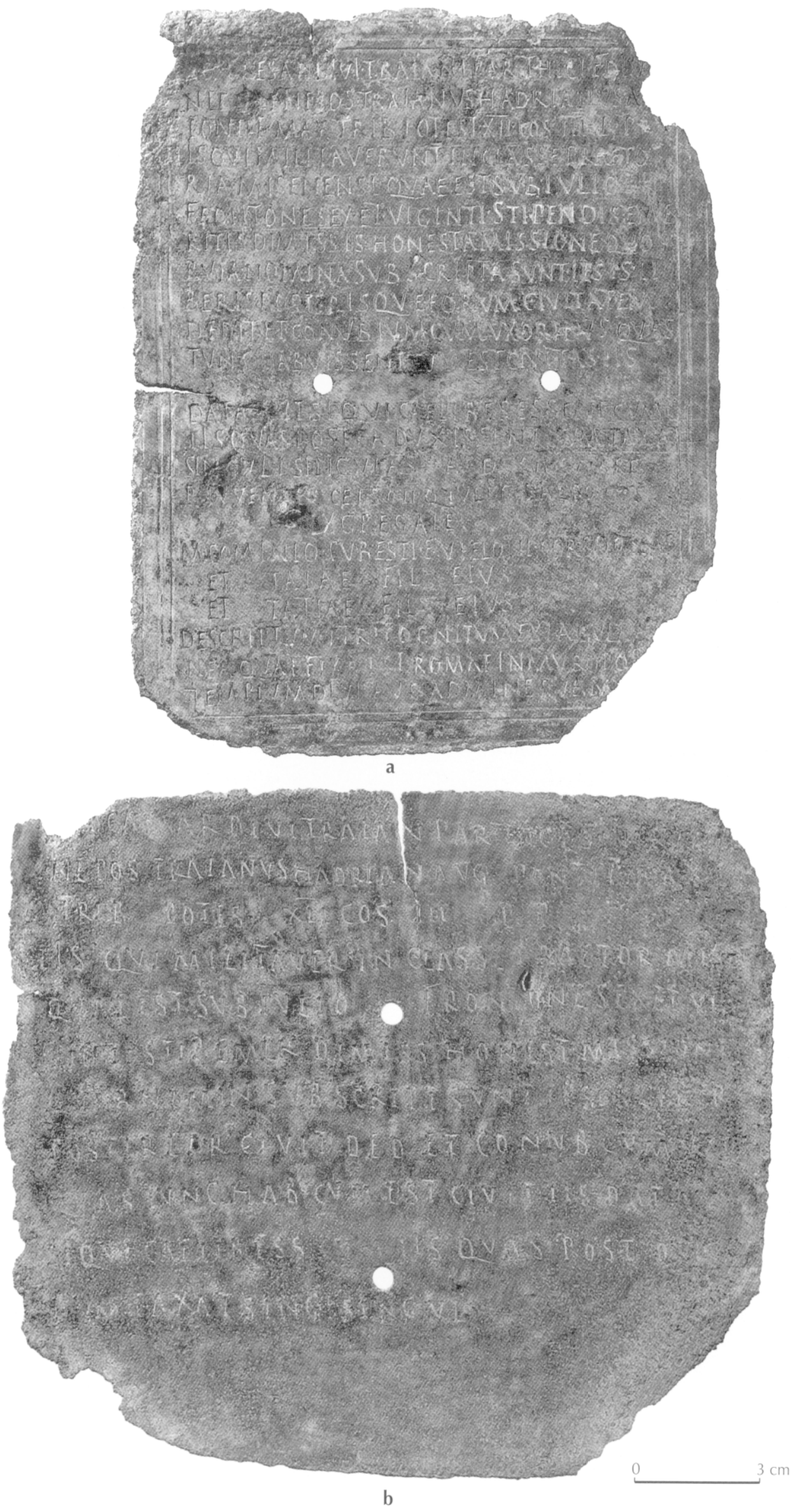

Fig. 1 - Diplôme militaire provenant d'Aléria (Haute-Corse), tahella I ;

$a$, extrinsecus ; $b$, intus (clichés M. Olivee, Service régional de l'archéologie Provence-Alpes-Côte d'Azur). 

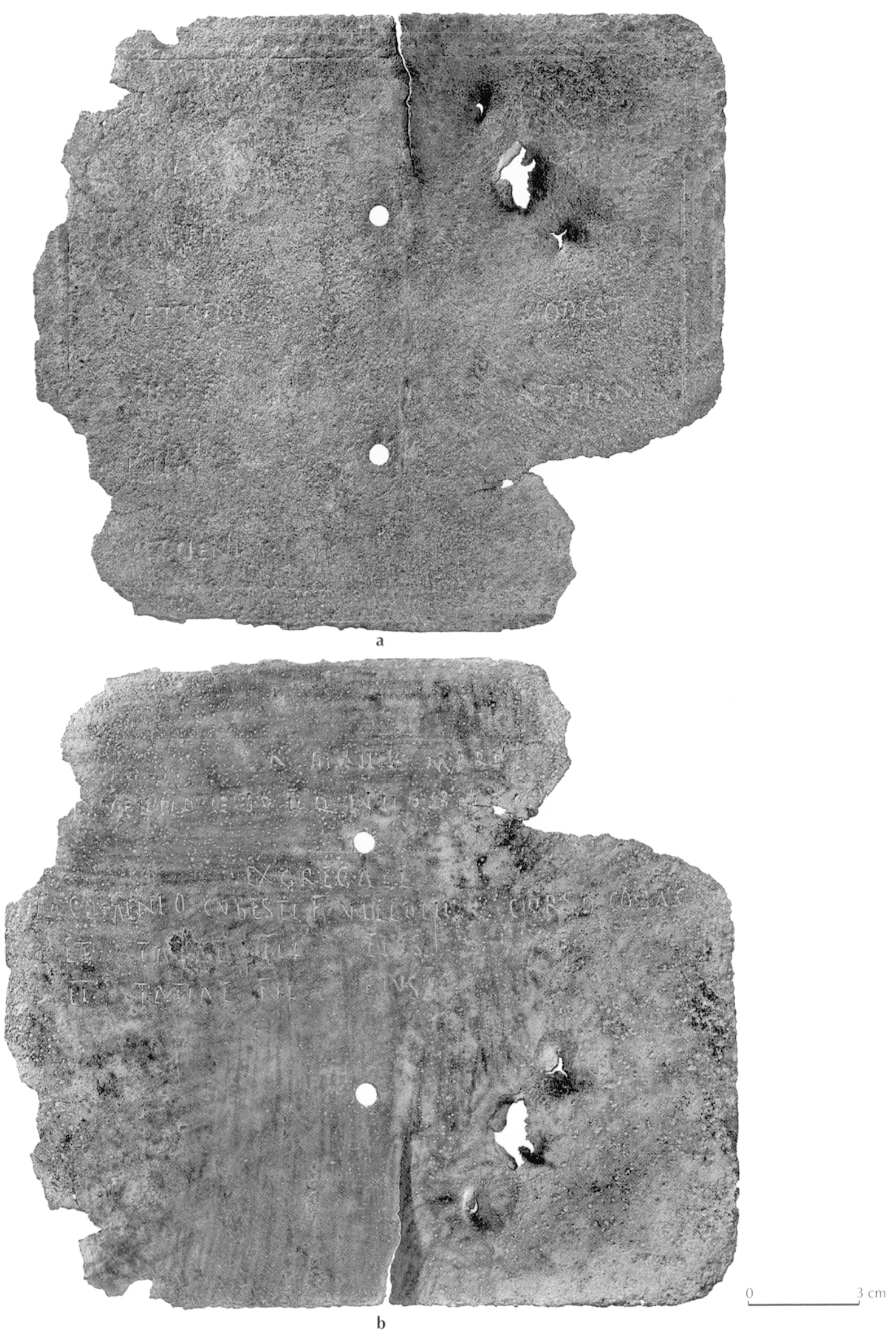

Fig. 2 - Diplôme militaire provenant d'Aléria (Haute-Corse), tabella II :

$a$, extrinsecus ; b, intus (clichés M. Olive, Service régional de l'archéologie Provence-Alpes-Côte d'Azur). 
(dr. et g. se référant à la face externe); poids $184 \mathrm{~g}$. Il manque l'angle inférieur dr. et ceux de gauche; les rebords sont rongés; il existe une cassure verticale d'environ $4,5 \mathrm{~cm}$ vers le milieu du côté supéricur ; trois coups ont perforé la surface dans le quadrant sup. g. ; la surface extérieure est oxydée.

\section{Extrinsecus (fig. 2a)}

Champ : 13,7 cm x 16,7 cm ; larg. du cadre : $1,2 \mathrm{~cm}$; h. des lettres : 0,3-0,5 cm ; interligne : 1,4-1,7 cm. Le texte est encadré par un simple filet.
[Ti.] $\operatorname{Iul}[i i]$
[C.] Caes $[i]$
Vrbani
Ti. Claudi
$[R]$ omani
C. Vettieni
$M[e n]$ andri

$\begin{array}{ll}\text { [L.]Attei } & \text { Atteiani } \\ \text { L.Pul[l]i } & {[\text { Verecundi] }} \\ \text { [C.]Vettieni } & \text { [He]rm[etis] }\end{array}$

$\check{5}$

Intus (fig. 2b)

H. des lettres : $0,3-0,5 \mathrm{~cm}$; interligne : 0,8-1,5 cm.

$A($ nte) di(em) $\overline{X I I} k$ (alendas) Mart(ias) [P.] Iuuentio Celso $\overline{I I}$ (. Iulio Balbo $[o(n) s($ ulibus $)]$.

$$
\text { ex gregale }
$$

M. Cominio Cubesti f(ilio) Vieloni corso cobas (io?)

5

el Taiae fill(iae) eius

et Taticue fil(iae) eius.

\section{TRADUCTION}

\section{TABELLA I}

\section{Extrinsecus}

L'empereur César Trajan Hadrien Auguste, fils du divin Trajan Parthique, petit-fils du divin Nerva, pontife maxime, dans sa XII ${ }^{\mathrm{C}}$ puissance tribunicienne, trois fois consul, père de la patrie, a octroyé, à ceux qui ont accompli leur service dans la flotte prétorienne de Misène, que commande Iulius Fronto, et qui, ayant mérité de recevoir 26 années de solde, ont été renvoyés avec un congé honorable, dont les noms suivent, à ceux-là, à leurs enfants et aux descendants de ceux-ci, la citoyenneté romaine et le droit d'épouser celles avec lesquelles ils vivaient au moment où la citoyenneté leur a été donnée, ou, s'ils n'avaient pas alors de femme, avec celles qu'ils prendraient pour épouses, en se bornant chacun à une seule.

(Fait) le douzième jour avant les calendes de mars sous le deuxième consulat de Publius Iuventius Celsus et le consulat de Quinctus Iulius Balbus.

À l'ancien grégal Marcus Cominius Viélon, fils de Cubestus (ou Cubestius), corse cobas(ius?), et à sa fille Taia et à sa fille Tatia.

Transcrit et retenu conforme à la table de bronze affichée au mur situé derrière le temple du divin Auguste près [de la statue] de Minerve à Rome.

\section{Intus}

L'empereur César Trajan Hadrien Auguste, fils du divin Trajan Parthique, petit-fils du divin Nerva, pontife maxime, dans sa XII ${ }^{e}$ puissance tribunicienne, trois fois consul, père de la patrie, a octroyé, à ceux qui ont accompli leur service dans la flotte prétorienne de Misène, que commande Iulius Fronto, et qui, ayant mérité de recevoir 26 années de solde, ont été renvoyés avec un congé honorable, dont les noms suivent, à ceux-là, à leurs enfants et aux descendants de ceux-ci, la citoyenneté romaine et le droit d'épouser celles avec lesquelles ils vivaient au moment où la citoyenneté leur a été donnée, ou, s'ils n'avaient pas alors de femme, avec celles qu'ils prendraient pour épouses, en se bornant chacun à une seule.

\section{TABELLA II}

\section{Extrinsecus}

[Ont signé comme témoins] Tibcrius Iulius Urbanus, Caius Caesius Romanus, Tiberius Claudius Menander, Caius Vettienus Modestus, Lucius Atteius Atteianus, Lucius Pullius Verecundus, Caius Vettienus Hermes.

\section{Intus}

(Fait) le douzième jour avant les calendes de mars sous le deuxième consulat de Publius Iuventius Celsus et le consulat de Quinctus Iulius Balbus.

À l'ancien grégal Marcus Cominius Viélon, fils de Cubestus, corse cobas(ius?), et à sa fille Taia et à sa fille Tatia.

\section{COMMENTAIRE}

La date de promulgation de la constitution nous est fournie par la titulature d'Hadrien - notamment par la mention de sa XII ${ }^{\mathrm{e}}$ tribunicia potestas - et se place entre le 10 décembre 127 et le 9 décembre 128 (Kienast, 1990, p. 129-130). Elle concerne les marins de la classis pratoria Misenensis ayant mérité de recevoir vingt-six années de solde ; la flotte était à ce moment-là sous les ordres de Iulius Fronto ${ }^{2}$. La présence en Corse, vraisemblablement à Aleria, d'un détachement de la classis praetoria Misenensis se fonde aussi bien sur les sources littéraires (Tacite, Histoires, II, 16, 2) qu'épigraphiques ${ }^{3}$. La flotte était jugée pour être "le moins prestigieux des corps de l'armée romaine, le moins rémunérateur et celui dont la durée d'engagement était la plus longue ${ }^{4}$.

2. Nous connaissons un Iulius Fronto préfet en $119: P I R^{2}, \mathrm{I}, 324$, p. 216. Pour Pflaum (1961, p. 1042) et Reddé (1986, p. 673), il ne s'agit pas du préfet de notre diplôme. Voir en outre, PME, I, 66 (I, 459 ; IV, 1604 ; V, 2140 ; VI, 38); Alföldy, 1986, p. 415.

3. Voir annexe, p. 289 et un catalogue des inscriptions trouvées en Corse ou bien concernant l'île, dans Zucca, 1996, p. 209-296 (Appendice epigrafica). Sur le détachement de la flotte de Misène à Aleria: ibid.. p. $182-187$ et Reddé, 1986, p. 207-211.

4. . [...] il meno prestigioso fra i corpi dell esercito romano, il meno retribuito e il piu lungo per anni di ferma "(Parma, 2002, p. 323 et notes). 
Le formulaire des droits octroyés est normal : il s'agit de la ciuitas et du conubium ${ }^{5}$. Suit la date de publication, avec l'indication du jour et du couple de consuls - $P$. Iuuentius Celsus II (PIR ${ }^{2}$ I, 882, p. 366 ; Degrassi, 1952, p. 37, n. 179) et Q. Iulius Balbus (PIR, I, 200, p. 152 ; Degrassi, 1952, p. 37, n. 179) - qui correspond au 18 février 129 . Le décalage entre les deux indications chronologiques correspond, comme l'a bien montré $\mathrm{W}$. Eck, à l'intervalle de temps entre la promulgation de la constitution et sa publication: " deux étapes différentes dans la préparation des constitutions " ${ }^{6}$. Il s'agit en général de quelques jours, mais il y a des cas où la différence atteint plusieurs mois (jusqu'à 10, cf. CIL, XVI, 38). En ce qui concerne le jour de publication de notre constitutio, il est utile de rappeler que le 17 et le 18 février avait lieu la célébration des fêtes des Quirinalia (RE, s.u. "Quirinus"). Ce choix - dies constitutionum datarum - n'est pas sans valeur : comme l'a bien souligné Dušanić : "la remise des diplomata était considérée par les Romains comme un acte cérémonial d'un intérêt plus étendu, qu'ils soient militaires ou civils " ${ }^{7}$, ce qui est confirmé par le choix des lieux d'affichage, sur lesquels nous reviendrons (infra, p. 284-285), qui « semblent avoir été sélectionnés dans un but plus vaste que celui simplement "pratique" " 8 .

Suit le nom du titulaire, M. Cominius, Cubesti f(ilius), Vielo, corsus cobas(ius ?) ${ }^{9}$, ancien grégal, qui transmet le droit de cité à ses filles Tatia et Taia: le nom de sa femme n'étant pas mentionné, soit il était veuf au moment de son congé, soit elle possédait déjà le droit de cité. Des tria nomina de sa formule onomastique, le gentilice a une très grande diffusion dans les provinces occidentales (OPEI, II, 70, s.u. "Cominius "), notamment dans la Cisalpine de l'ouest (Gregori, 1990, p. 59-61). Le cognomen Vielo (?) est vraisemblablement le nom originel du personnage comportant, comme celui de son père, un seul élément; aussi bien l'un que l'autre sont, à notre connaissance, des hapax legomena.

L'indication de l'origo est tout à fait courante dans les diplômes militaires et elle est bien attestée parmi les classiarii (Parma, 2002, p. 326, tabl. I) ; notre personnage se dit donc corsus, comme c'est le cas de cinq autres marins de la flotte de Misène (Parma, 2002 et annexe, infra, p. 289), de quatre autres de celle de Ravenne (Giacomini, 1990 et annexe, infra, p. 289) et de deux autres dont la classis d'appartenance nous est inconnue (annexe, infra, p. 289). Ce qui pose problème est l'adjectif cobas(ius?), qui semble ensuite spécifier la provenance de notre personnage, comme c'est le cas pour d'autres

5. Sur les privilèges octroyés au moment du congé et sur leur développement, voir Wolff, 1986 ; sur le formulaire et ses variations, voir $R M I$, III, appendix 3,341 .

6. " $[\ldots]$ zzwei verschiedene Stadien bei der Ausfertigung der Konstitutionen " (Eck, 2002, p. 258).

7. " $[\ldots]$ the issue of diplomata was regarded as a somenhat ceremonial act of wider interest for the Romans, both the army and the civilians. "(Dušanić, 1985 , p. 233).

8. "[ [...] seem to have been selected with a purpose beyond the "practical" one. " (Dušanić, 1985, p. 233).

9. Sur l'onomastique des titulaires des diplômes, voir Mócsy, 1986. militaires, parmi lesquels les marins corses qui nous sont jusqu'ici connus. $M$. Numisius Saionis $f$. Nomasius, lui aussi ancien grégal, est mentionné sur un diplôme (CIL, XVI, $74=\mathrm{V}, 4091$ ) dérivant de la même constitutio, dont le texte est donc identique à celui d'Aleria. Ce personnage est dit corsus uinac (sc. Vinacinus: plutôt de Ovievíkıv voir Ptolémée, Guide géographique, 3, 2, 7 que Oủavaknvós, id., ibid., 3, 2, 6). Ce dernier diplôme fut trouvé près de Crémone avec un autre (CIL, XVI, $102=\mathrm{V}, 4092)$, datable entre 149 et 153 , mentionnant encore une fois un ancien grégal appartenant à un peuple de la Corse sud-orientale : $L$. Valerius Cainensis f. Tarvius, opinus ex Corsica (Ptolémée, Guide géographique, 3, 2, 7 : 'O L'énonciation de la provenance géographique se retrouve dans moins de la moitié des inscriptions des classiarii qui nous sont parvenues, notamment sur 131 des 233 de la classis praetoria Rauennatis et sur 288 des 620 de la Misenensis (Parma, 2002, p. 325-326). À partir du III' s. l'origo est en outre précisée par l'indication du uicus (Weiss, 2000).

Le problème que pose cette nouvelle inscription est lié au fait qu'aucune des sources mentionnant les diverses cités, les toponymes et les peuplades de la Corse ne fait mention de cobas ou de quelque terme qui aurait pu subir une contraction ou une abréviation similaire ${ }^{11}$. Ni cobas ni - en considérant le patronyme comme faisant allusion à un toponyme - cubest ne trouvent une correspondance dans la toponymie de la Corse contemporaine.

En ce qui concerne les filles, il faut en premier lieu observer qu'il peut s'agir de la duplication d'un seul nom ${ }^{12}$; leur formule onomastique se limite au gentilice, différent de celui du père, utilisé comme cognomen.

L'original de la constitution dont notre diplôme est un extrait avait été affiché à Rome sur le mur derrière le temple du divin Auguste près de la statue de Minerve, comme c'est le cas pour tous ces documents à partir de 90 apr. J.-C. ${ }^{13}$. En effet il serait plus exact, en suivant Dušanić, de parler de "deuxièmes originaux ", les " premiers " étant vraisemblablement conservés à l'intérieur du tabularium publicum (Dušanić, 1984, p. 91, n. 1). La valeur de l'affichage a été déjà soulignée à propos des jours qui étaient choisis pour cet acte ${ }^{14}$ et il faut ajouter la valeur de propagande des loci choisis, ainsi que du "système " des diplômes, qui serait confirmée - comme le montre de façon convaincante S. Dušanić (1984, p. 113-114) - par les changements successifs qui eurent lieu dans le temps, jusqu'au choix

10. Nous préférons, sur la base des mentions d'appartenance figurant dans les diplômes militaires, voir ici l'adjectif plutôt que la mention de la cité (Ptolémée, Opinon, 3, 2, 8), qui aurait été précédée par ex.

11. Notamment Ptoléméc, Guide géographique, 3, 2 ; Pline l'Ancien, Histoire naturelle, III, 80 ; Itinéraire antonin, 85-86, 1. À notre connaissance, une seule cité possède un nom très proche, mais elle se trouve près d'Igilgili, en Maurétanie césarienne: il s'agit du Coba municipium mentionné dans l'Itinéraire antonin (18), correspondant à la Ziama actuelle.

12. C'est à M. I.. Caldelli que je dois cette remarque, dont je la remercie.

13. Sur les lieux d’affichage, voir Dušanić, 1984 ; pour les plus anciens diplômes de classiarii de la flotte de Misc̀ne, Forni, 1986, p. 307-309.

14. Dušanic, 1985 ; voir aussi supra, p. 284. 
définitif du murus post templum diui Augusti ad Mineruam, qui reste le seul à partir de 9015 .

Les témoins, au nombre de sept, figurent sur la face externe de la tabella II. Leurs noms sont connus par plusieurs autres diplômes et leurs services se situent dans une période allant de 78 (L. Pullius Verecundus) à 129 ( $I$. Atteius Atteianus) ; ils figurent ensemble sur l'autre diplôme (CII, XVI, 74) se référant à la constitutio, dont il a été question. L'activité de I. Pullius Verecundus a une durée plutôt exceptionnelle (52 ans), qui pourrait suggérer l'hypothèse que l'on est en présence de deux personnages homonymes, vraisemblablement père et fils. Dans un premier temps, les témoins qui assuraient la conformité du diplôme à l'original du décret étaient des camarades ou bien des compatriotes du bénéficiaire ; ensuite, à partir de 74, ces documents sont signés par sept témoins, toujours les mêmes pendant une certainc période, qui semblent avoir exercé cette profession dans un bureau à Rome. Ils apparaissent aussi bien sur les diplômes des auxiliarii que sur ceux des classiarii. Leurs noms se suivent d'abord sans un ordre défini, ensuite la liste est dressée suivant leur ancienneté (Morris, Roxan, 1977 ; Saddington, 1997).

Ce diplôme représente donc un témoignage ultérieur de la constitutio déjà connue par le document de Crémone (CIL, XVI, 74) ; il nous donne, en outre, un nouveau toponyme ou, micux, l'indication d'un toponyme jusqu'ici inconnu, voire le nom d'une peuplade, ainsi que des données nouvelles sur l'onomastique indigène de la Corse.

\section{L'INSCRIPTION EN GREC}

Cette inscription (fig. 3) fut trouvée lors d'une opération d'archéologie préventive menée sur le versant oriental du plateau d'Aléria, à la périphérie du hameau actuel, afin "d'évaluer le risque archéologique en amont de la construc-

15. Sa construction remontait à l'époque julio-claudienne: après sa destruction à causc d'un incendic en 69 ou en 80 , il fut reconstruit par Domitien et sa dédicace cut lieu en $890 u 90$ (LTUR, I, s.u. : Augustus, diuus, templum (nouum) ; aedes [M. Torelli], p. 145-146). À la même époque les constitutions commencent à être affichées au mur derrière ce temple. Une nouvelle hypothèse fait état d'une reconstruction topographique tout à fait différente : Ic templum diui Augusti, ou mieux les divers réaménagements qu'il subit dans le temps auraient donné comme résultat la plateia de la prétenduc Domus Tiberiana (Cecamore, 2002, p. 202). Le podium en opus caementicium découvert au-dessous de l'hôpital de Santa Maria della Consolazione, d'autre part, serait à identifier arec le temple de Minerua et il aurait été le support pour l'affichage des tabulae honestae missionis (La Rocca, 2002, p. 6-7). Si le mur est à placer sur le Palatin, le transfert du Capitole à celui-ci n'est pas sans signification politique: il s'agit d'un passage du tabularium publicum et du Capitole, qui ont gardé leur valeur de siège privilégié pour toute la période julio-claudienne, au tabularium principis sur le Palatium; un changement dont la valcur politique est significative (Mastino, 1993). 
Le texte est gravé sur une dalle de marbre à gros grain $(\mathrm{h}$. : $29,5 \mathrm{~cm}$; larg. : $36,5 \mathrm{~cm}$; ép. : $3 \mathrm{~cm})$; la hauteur des lettres varie entre $1 \mathrm{~cm} \mathrm{(1.2)} \mathrm{et} 1,5-1,6 \mathrm{~cm}(1.4)$. Les lettres initiales $\Theta$, $\mathrm{K}, \mathrm{X}$ sont beaucoup plus grandes $(2-3 \mathrm{~cm})$. On remarque quelques imprécisions dans la gravure des lettres : parfois le trait interne de l'alpha est absent; les kappa sont tous différents les uns des autres; des lettres surmontent la ligne d'écriture (le kappa de la l. 4, le tau de la 1. 5, le psi de la 1. 6). Epsilon, sigma et omega sont lunaires, le $m u$ tend à l'italique. À la cinquième

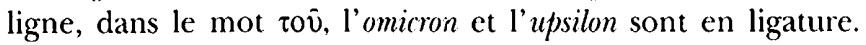
Deux signes en forme de croix de saint André, dont la fonction n'est pas claire, se trouvent l'un en haut, près de l'angle droit, l'autre en bas, au centre de la dalle.

Il est possible de dater ce texte, sur la base de la paléographie et de l'orthographe, au plus tôt de la fin du III's. apr. J.-C.

\section{TEXTE INÉDIT}

\author{
$\Theta(\varepsilon \circ \hat{\imath} \varsigma) \mathrm{K}(\alpha \tau \alpha) \mathrm{X}($ Oovioı $\zeta)$

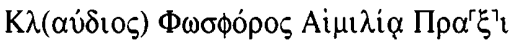

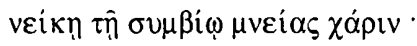

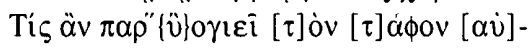

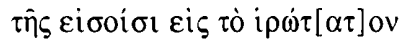 \\ $\tau \alpha \mu \hat{i}$ ov $\tau$ oิ $\pi \rho \circ \sigma \tau \varepsilon i \mu(o v) * \alpha \cdot \dot{\omega} \delta \varepsilon$

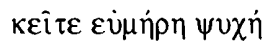

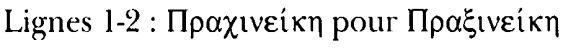

\section{«Aux Dieux Souterrains}

Klaudios Phosphoros à sa femme, Aimilia Praxinike, en souvenir.

en forme de cuvette (cf. tranchée suivante) prenant en comptc peutêtre la partie plane de l'actuel parking (niveau sup. à la parcelle diagnostiquée). L'accumulation, en alternance, d'une séric de couches limoneuses organiques et de lits de petits graviers tendrait à prouver qu'il y a eu, sur ce palier intermédiaire du vallon, une retenue d'eau stagnante, sorte de zone marécageuse, connaissant par moment des variations d'activité avec dépôts de type fluviatile. L'eau devait ensuite dévaler en contrebas, au creux du vallon.

"Sur ce remblai repose une couche (90) hétérogène comportant de nombreux matériaux concassés (briques, tuiles, céramiques...) en petits morceaux. ("'est dans ce niveau (idem 97 de la tranchée ${ }^{\circ} 7$ ) qu'ont été installées les sépultures. I a surface de cette couche a dû servir de niveau de circulation. 4 structures en briques liées au mortier $(39,40,41$ et 42), pouvant être assimilées à des tombes, ont pu être localisées dans cette tranchée (deux cortaines: 40 et 41). Pour des raisons dues à la nature même du diagnostic il n’a pas été envisagé d'entreprendre la fouille de l'une d'entre elles. Un remblai (91) a été enregistré à l'aplomb de la structure 39. Il pourrait s'agir d'un petit dôme de terre, aérien, recouvrant la tombe

"L'ensemble des structures est recouvert d'un remblai (36) hétérogène, mélange de colluviomnement et de terres végétales. ("est dans ce remblai, lors du décaissage des parties supérieures de la tranchée, qu'une plaque de marbre à vocation funcraire a pu c̀tre, sous les assauts de la pelle mécanique, in extremis sauvéc de l'oubli. Cette pièce comporte des inscriptions en gree (deuxième du genre trouvée à Aléria, cf. Inventaire). Il s'agit bien d'une dédicace appartenant à un contexte bas limpire. ". Dans l'inventaire du mobilice de l'LS 36 [p. 51] il est, en effet, question d'une scule inscription.
Si quelqu'un ouvre le tombeau de celle-ci, il versera au fisc très saint, comme amende, 1000 deniers ${ }^{19}$. Ci-gît une âme heureuse! »

Le texte présente des irrégularités dans l'orthographe, dues à des phénomènes d'itacisme, qui nous font pencher pour une datation non antérieure au milieu du $\mathrm{III}^{\mathrm{c}}$ s. apr. J.-C., ce qui est d'ailleurs en accord avec les caractéristiques paléographiques dont il a été question ; quelques-unes de ces irrégularités sont

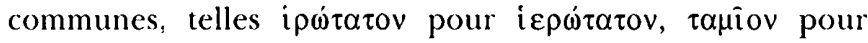

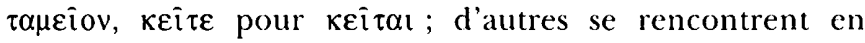
revanche moins fréquemment. Dans le cas de eijuńp au lieu de $\varepsilon \ddot{\mu} \mu$ otpos (1. 6), la diphtongue oi, qui se prononce toujours iota, est rendue par $\eta^{20}$ et, en ce qui concerne la désinence, nous trouvons une terminaison féminine en eta, au lieu du maintien de la désinence normale en - -5 , s'agissant d'un adjectif à deux terminaisons ${ }^{21}$. La forme $\pi \alpha \rho v o \gamma ı \varepsilon \hat{\imath}$ de la ligne 3 présente un problème qui ne se limite pas à la graphie : les verbes qu'il aurait été normal de trouver dans ce contexte sont $\pi \alpha \rho \alpha v o i \gamma \omega$ ou bien $\pi \alpha \rho o i \gamma \omega$, qui ont un futur sigmatique; ici la désinence - $\iota \iota$ ne peut être expliquée que comme une variante d'un futur de type attique dérivant d'un présent du type oi $\gamma^{\prime} \zeta_{\omega} / \dot{o} \gamma^{\prime} \zeta_{\omega}$

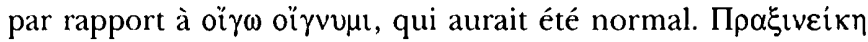

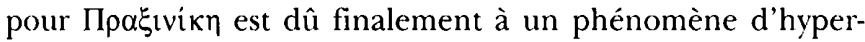
correction ; il faut aussi remarquer, dans ce nom, que la forme du ksi grec correspond apparemment à celle du chi, mais il est en revanche à interpréter, d'après nous, comme étant une confusion avec la forme du $x$ latin.

Pour ce qui est de la lecture, le texte ne présente aucun problème : même la fracture entre les lignes 3 et 4 peut ĉtre intégrée sans difficulté, sur la base des formulaires et des traces de lettres qui subsistent. Une seule incertitude demeure pour le début de la ligne 4, à cause de l'absence totale du trait transversal du $t a u$, mais il nous semble que av̀in̄ soit la seule lecture possible.

La dédicace aux Dieux Souterrains (qui, dans ce cas, est indiquée par $\Theta \mathrm{KX}$, au lieu du $\Theta \mathrm{K}$ habituel, en divisant donc l'adjectif $\kappa \alpha \tau \alpha \chi \theta$ óvios dans ses deux composantes ${ }^{22}$ ) atteste sans aucun doute qu'il s'agit d'un texte funéraire. La défunte est une certaine Aimilia Praxinike et l'inscription est apposée par son mari Klaudios Phosphoros.

Le formulaire des deux premières lignes est tout à fait normal, la partie qui suit est moins commune: on y trouve la

19. Il est aussi possible d'interpréter le $m u$, qui est d'ailleurs écrit audessous du signe du denier, comme indiquant la dizaine de milliers, qui serait ensuite spécifiée par l'alpha comme multiplicateur; c'est-à-dire 10000 deniers: voir par exemple Strubbe, 1997, n. 96 ct 145 d'Aphrodisias. Quant à la possibilité d'un montant aussi élevé pour une amende, voir infra.

20. Ces différentes graphies sont dues à des phénomènes de prononciation de l'époque impériale avancée, et sont donc très fréquentes dans les inscriptions chrétiennes (Creaghan, Raubitschek, 1947. p. $18-21)$.

21. Nous ne sommes pas d'accord avec Derda (1986), pour qui le terme

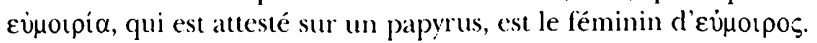

22. Cet usage, même s'il est plus rare, est néanmoins largement attestć : voir par exemple, pour l'Italic, les inscriptions grecques de Rome (Moretti ed., I(;UR, II, Index). 
mention d'une amende, prévue au cas où quelqu'un ouvrirait le tombeau, contre la volonté du dédicant. Les amendes funéraires sont parfois associées à de véritables malédictions, dans la catégorie des $\dot{\alpha} \rho \alpha i$, à savoir des inscriptions de malédiction contre les violations du sépulcre ${ }^{23}$. L'usage de graver sur les tombeaux des inscriptions ayant comme but celui d'en sauvegarder l'intégrité avant même la propriété est bien attesté dans le monde gréco-oriental (surtout en Phrygie, en Carie, en Lydie et en Lycie) à partir de l'époque hellénistique. La plupart de ces documents prévoient des formes diverses de malédiction envers celui qui viole le tombeau d'une façon ou d'une autre ${ }^{24}$ (la terre ne donnera plus de fruits ; sa progéniture sera détruite ; il sera frappé par les disgrâces...). Il y a, en outre, des cas où le paiement d'une amende de la part du violeur est prévue ${ }^{25}$. Des éléments constants sont présents dans ces formules : l'ouverture

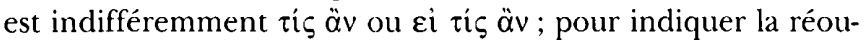
verture du tombeau, on emploie en général le verbe $\dot{\alpha} v o i \gamma \omega$ ou, plus rarement, $\pi \alpha \rho \alpha$ voi $\gamma \omega$ qui signifie plus spécifiquement " ouvrir en partie ", " entrouvrir " ${ }^{26}$. En revanche, nous ne connaissons pas d'autres attestations épigraphiques de la forme $\pi \alpha \rho o i \gamma \omega$, dont la signification équivaut à $\pi \alpha \rho \alpha v o i \gamma \omega$, qui se trouve par contre dans le lexique de la tragédie et de la comédie attiques (Euripide, Iphigénie en Aulide, 857; Aristophane, Ia Paix, 30). Quant au verbe $\varepsilon i \sigma \phi \dot{\varepsilon} \rho \omega$, qui est employé ici pour indiquer l'acte du paiement, on peut remarquer que, tout en étant le terme spécifique dans le lexique des documents de type financier, il semble moins souvent attesté dans le formulaire des amendes funéraires ${ }^{2 i}$. Les verbes le plus souvent employés

23. Toutes les attestations de ce type d'inscriptions, groupées selon la typologie du formulaire, ont été récemment recueillies par J. Strubbe (1997).

24. 'A $\alpha v i \zeta \varepsilon$ ' de l'inscription ou du relief est considéré lui aussi comme une violation grave du tombeau (Strubbe, 1997, n. 15, 148, 369, etc.).

25. Le phénomène des amendes funéraires, notamment en milieu romain, a été étudié à plusieurs reprises. Un recueil utile de ce matériel, effectué sur la base du montant de l'amende, se trouve dans Rossi, 1975; une table ronde a été consacrée à ce sujet à l'École française de Rome (Collectif, 2004), au cours de laquelle il a été question de faire le point de la situation, sur la base des documents aussi bien latins que grecs. On renvoie donc aux actes de cette table ronde et, parmi les interventions, à celle de T. Ritti en particulier, qui a présenté les nombreuses attestations présentes dans les inscriptions de Hierapolis de Phrygic. Il reste nćanmoins des aspects qui n'ont pas été complètement éclaircis dans leurs diverses facettes d'ordre juridique et religieux ct dans leur matrice - à chercher selon certains en milieu grec, justement dans les inscriptions de malédiction d'Asie Mineure, selon d'autres dans le cadre du droit romain. On peut remarquer néanmoins, en ce qui concerne le monde grec, qu'en Phrygie, la région qui présente le plus grand nombre d'inscriptions de malédiction (Strubbe, 1997, n. 156-297), huit seulement font état d'une amende (n. 228-229, 244, 248, 285, 289, 291-292) et, en tout cas, les défunts sont des citoyens romains ou bien font partie de la communauté juive.

26. Voir Strubbe, $1997: 50$ cas pour le premier, un seul pour le deuxième, de Diocésarée en Cilicie (n. 384).

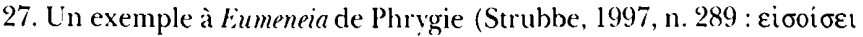
$\tau \hat{\omega} \phi i \sigma \kappa \omega)$; trois à Ephèse (Merkelbach, Nollé eds, 1980, VI, n. 2435) :

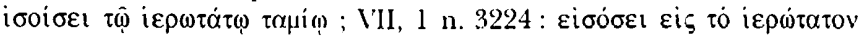

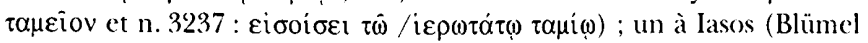

sont : $\delta \mathrm{i} \delta \omega \mu \mathrm{l} / \dot{\alpha} \pi \circ \delta i \delta \omega \mu \mathrm{l}(\delta \omega \dot{\omega} \sigma \varepsilon)^{28}$ ou $\tau \dot{i} \theta \eta \mu \mathrm{r}(\theta \dot{\eta} \sigma \varepsilon \mathrm{l})^{29}$ ou

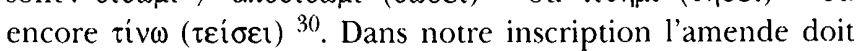

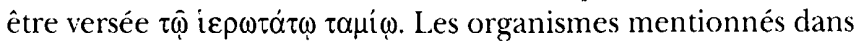
les inscriptions grecques sont généralement deux : фiбко ou $\tau \alpha \mu \varepsilon i v^{31}$, auxquels s'ajoutent parfois "la ville " 32 , " la Boulé " ou " la Gerousia" " ${ }^{33}$, dans d'autres cas encore un sanctuaire ou,

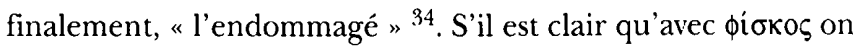
fait allusion à la caisse personnelle de l'empereur (et en fait

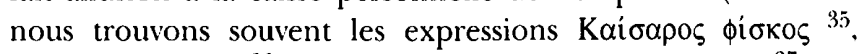

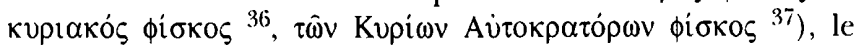
terme tameion, qui est fréquemment défini hieron ou bien hierotaton, a donné lieu dans le temps à la formulation d'hypothèses diverses (caisse d'un temple, trésor citadin) ${ }^{38}$, mais en réalité, comme on l'a vu, l'un et l'autre, lorsqu'ils apparaissent dans les inscriptions, sont indiqués de façon spécifique. Comme certaines inscriptions nous le révèlent, même le terme tameion faisait allusion à la caisse impériale ${ }^{39}$.

En ce qui concerne le montant de l'amende, comme on l'a $\mathrm{vu}^{40}$, en interprétant le mu qui se trouve au-dessous du signe du denarius comme l'initiale des termes $\mu(\text { v́pırol) ou } \mu \text { (vpı́́s })^{41}$,

ed., 1985, II, n. 378) ; un à Smyrne (Petyl ed., 1982, I, n. 202), etc. Dans les inscriptions de Mylasa, on remarque une division nette entre $\varepsilon i \sigma \emptyset \varepsilon \dot{\varepsilon} \omega$, qui est employé dans les cas de paiements ordinaires et de contributions

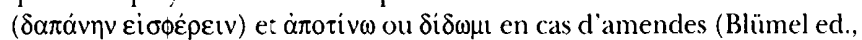
1985, I, respectivement, n. 118 , l. 9 et n. 444 et 455 ).

28. Strubbe, 1997 , n. 9, 32, 140-142, 145, 154, 345, etc.

29. Strubbe, 1997, n. 228, 291-292, 303, 358.

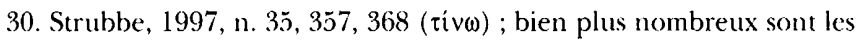
exemples avec $\dot{\alpha} \pi 0 \tau i v(\omega$.

31. Le premier, quoiqu'il s'agisse de la translittération du terme latin, est employé régulièrement dans les documents grecs; en revanche,

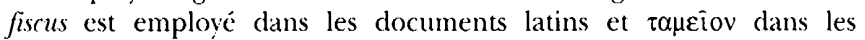
documents grecs seulement à Concordia (Rossi, 1975, p. 127).

32. La polis et le fisc sont associés à Nicomédic (Petzl ed., 1982, p. X, n. $17 ; I G R, I V, 1460)$ et la polis et le hierotaton tameion à Alexandrie de Troade (IGR, IV, 245). La seule polis est mentionnée, par excmple, seulement dans les inscriptions de Philippes (Rossi, 1975, p. 128).

33. La kratiste Boulé est mentionnée par exemple dans une inscription d'Aphrodisias (Strubbe, 1997, n. 92) et la Gerousia à Smyrne (Strubbe, 1997, n. 27).

34. Le sanctuaire d'Artémis est mentionné dans les amendes de Bargylia (Strubbe, 1997, n. 75) et celui de \%eus Solymeus est associé à la Boulé sacréc à Termessos (Strubbe, 1997, n. 326); à Aphrodisias il est fait mention, dans un cas, de l'usage qu'il faudra faire de l'argent : $\varepsilon i \zeta \tau \imath \mu \dot{\alpha} \varsigma$ $\tau \bar{\omega} v \Sigma \varepsilon \beta \alpha \sigma \tau \bar{\omega} v$ : Strubbe, 1997, n. 101. À Hiérapolis de Phrygie, enfin, dans un cas on prévoit le versement de 2500 deniers au fisc, autant à la Boulé et autant à l'offensé (Strubbe, 1997, n. 285).

35. Strubbe, 1997, n. 32 (Téos) et n. 106 (Aphrodisias).

36. Strubbe, 1997, n. 90 (Aphrodisias).

37. Strubbe, 1997, n. 303 (Pisidie).

38. Pour ces hypothèses, voir Rossi, 1975, p. 135̄-137.

39. Ce serait prouvé par des textes où l'on mentionne le iepótatov

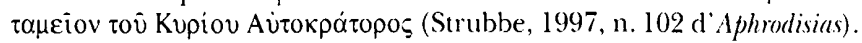
40. Voir supra, note 19, p. 286.

41. Ainsi, par exemple, à Éphèse on retrouve dans un cas " $\mu$ úpı $\alpha$ et dans un autre $\delta \eta v(\dot{\alpha} \rho t \alpha) \mu(\dot{\rho} \rho \iota) \alpha^{\prime}$ (.Merkclbach, Nollé eds, 1980 , respectivement $n$. 2435 et $2299 \mathrm{~b})$. 
nous serions en présence d'unc amende de 10000 et non de 1000 deniers. Il est vrai que le montant des amendes est en movenne de 250 ou 500 deniers, mais à l'époque impériale avancée, peut-être aussi à cause des dévaluations, nous trouvons des sommes bien plus élevées, jusqu'à 50000 denicrs : notre somme de 10000 deniers serait elle aussi, par conséquent, tout à fait acceptable ${ }^{42}$.

En résumant, donc, l'inscription est poscé sur la tombe d'une femme, clle est dédiée par le mari de celle-ci ct présente, dans son formulaire et dans son contenu, des éléments qui semblent en rapport avec l'Asie Mineure. Quant aux raisons de la présence d'un Grec en Corse à l'époque impériale, nous pensons qu'elles sont à rechercher essentiellement au scin de deux milieux: celui des affranchis impériaux et celui des marins de la flotte de Misène.

Un détachement de la flotte de Misène fut installé dans le port d'Aleria, à partir du règne d'Auguste ; cette flotte joua un rôle important par rapport à l'île pendant le conflit entre Othon et Vitellius. Son commandant était à l'époque Claudius Pyrichus, certainement un affranchi impérial, grec, originaire vraisemblablement d'Asie Mineure, comme la plupart des militaires de la flotte de Misène; comme l'était probablement Klaudios Phosphoros ${ }^{43}$. I a plupart des Crrecs dont le gentilice est Klaudios, ayant obtenu le droit de cité par Claude ou Néron,

42. Il faut considérer, en outre, qu'il n'est pas aisé de comprendre comment pouvaient se faire un contrôle réel et la perception de l'amende, ce qui a fait formuler l'hypothèse qu'elle pouvait n'avoir qu'un but purement dissuasif.

43. Sur la flotte de Misène, outre Chapot, 1896, classique, voir Starr, 1960. Dans ke tableau dressé par Chapot (1896, p. 186) sur les 200 classarii qui ćtaient comus à l'époque, plus de $30 \%$ étaient des Grecs et des Gréco-orientaux (parmi lesquels les Ciliciens étaient les plus nombreux) et ce pourcentage atteignait $60 \%$ en comprenant les Alexandrins, les Egyptiens et les I ibyens. se trouvent en Phrygie, en Ionie et en Carie ${ }^{44}$; notre personnage pourrait donc être un oriental descendant d'une famille à qui un de ces deux empereurs avait octrové le droit de cité, ou bien pouvait ĉtre le descendant d'un affranchi des Claudii.

Quant à l'élément proprement grec du nom, $Ф \omega \sigma \phi o ́ p o s$, il n'est pas attesté avant l'époque impériale ${ }^{45}$; on en connaît plusicurs à Rome dans les inscriptions latines qui mentionnent surtout des esclaves et des affranchis ${ }^{46}$, ainsi qu'un cas dans

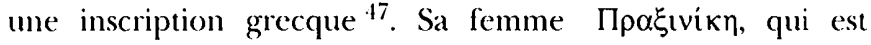
sûrement une affranchie de la gens Aemilia, a, elle aussi, un nom peu commun, surtout au féminin ${ }^{48}$. On ne retrouve qu'une seule attestation de la formule finale " ci-gît une âme

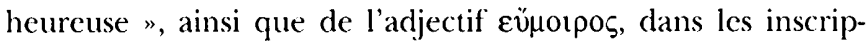
tions païennes de Rome ${ }^{49}$. L'adjectif est mieux attesté dans les inscriptions chrétiennes, notamment orientales, mais il manque dans notre texte une allusion chrétienne et le caractère païen est confirmé par la mention des dieux Mânes ; cet adjectif pourrait donc s'expliquer tout simplement par l'origine gréco-orientale présumée de notre personnage.

44. Voir Holtheide, 1983, p. 55 sq\%. et p. $486-487$ pour la liste des localités et les pourcentages d'attestations.

45. Fraser I : un exemple de Chypre et un de Ténos ; Fraser II contient plusicurs exemples d'Athènes; Fraser IIIA signale des cas de Corynthe, Sparte et, dans la forme latine, de Baies, Pompéi, Herculanum et Salerne.

46. Solin (1982, I, p. 385) signale 31 cas, dont 16 concernent sûrement des esclaves et des affranchis (Solin, 1999, II, p. 304).

47. Moretti ed., IGUR, I, 160, IIIA, ligne 36. D'autre part, la mobilité des Grecs au sein de l'Fmpire est largement attestéc, notamment pour Rome, Ostic el la Campanie.

48. On en connaît un scul exemple, celui de Messène, dans Fraser, IILA. 49. Morctti ed., IGUR, II, 717 , avec la bibliographic concernant cet usage dans les inscriptions chrétiennes. 


\section{ANNEXE}

\section{LES CLASSIARII}

\section{LES CLASSLARII CORSES DE LA FLOTTE DE MISÈNE}

M. Cominius Cubesti f(ilius) Vielo, gregalis, corsus coba: (territoire d'Aleria), 18 février 129.

M. Numisius Saionis f. Nomasius, gregalis, corsus uinac(inus?) : CII., XVI, 74 (territoire de Crémone), 18 février 129.

Cassius Albanus, nat(ione) cors( $u s)$ : Al: 1979, 166 (Misenum), $\mathrm{II}^{\mathrm{e}}$ s. apr. J.-C.

Cattius Viator, nat(ione) cors(us) : (.IL, X, 3562 (Misenum), Il' s. apr. J.-C.

T. Dinnius (eler; centurio, nat(ione) corsus: (IL, X, 3572 (Misenum), $\mathrm{II}^{\mathrm{e}}$ s. apr. J.-C.

C. Valeni[ius---]nus, miles, natione corsus: $A E, 1939,227$ (Seleucia de Pićrie), II" s. apr. J.-C.

\section{LES CLASSIARII CORSES DE LA FLOTTE DE RAVENNE}

I. Numisius Liberalis, miles, nat(ione) cursican(us) : (III, II, 4063 (Dertosa), II" s. apr. J.-C.

M. Marius Nepos, miles, natione cor(sus): $A E, 1929,140$ (Ostic), II's. apr. J.-C.

C. Titius Caelianus, optio, $n[$ at(ione) cor $]$ sicanus: CII, XI, 6741 (Ravenne).

L. Vicerius Tarsa, nal(ione) corsi(canus): CIL, XI, 109 (Ravenne).

\section{LES CLASSIARII CORSES DE FLOTTE INDÉTERMINÉE}

I. Valerius Cainensis f. Taruius, gregalis, Ofpimo ex Cors(ira) : CIL, XVI, 102 (territoire de Crémone), 24 décembre 153.

Anonyme, na(tione) corsus: (II, VI, 3172 (provenance inconnue).

\section{LES AUTRES CLASSIARII ATTESTÉS EN CORSE}

Baslel Turbelli f. Gallinaria Sarniensis, cl. Misenensis: (II, XVI, 16 (Algajola), 5 avril 71.

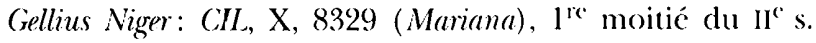
apr. J.-C.?

C. Caninius (jermanus, centurio, cl. Rauennatis: II. (SN, 14, II" s. apr. J.-C.

Iulius Mum[mius], scriba, cl. Misenensis: AE, 1965, 145 (Aleria), II"s. apr. J.-C.

Apronius Felix, miles et praeco praefecti, cl. Misenensis: Ephemeris Epigraphica, VIII, p. 200, 800 (Aleria) III' s. apr. J.-C.

Anonyme, [optio?] nauali $[$ or $(u m)]: A L, 1968,284$ (Aleria).

\section{INDEX DES NOMS CORSES INDIGÈNES}

Cainensis (CIL, XVI, 102).

Cubestus/Cubestius (voir supra, p. 280-285).

Eunus (CIL, X, 8038).

Lasemo (CIL, X, 8038).

Saio (CII, XVI, 74).

Tarsa (CII., XI, 109).

Tomasus/Tomasius (CII, X, 8038).

Vielo (voir supra, p. 280-285). 


\section{BIBLIOGRAPHIE}

Abréviations
$A E$
BEFAR
CII.
$I C R$
IGUR
Inschrift. von Ephesos
Inschrift. von Iasos
Inschrift. von Mylasa
Inschrift. vm Smyrna
ITCR
OPELL
$P I R^{2}$
$P M E$
$R E$
$R M D$
$Z P E$

ALFÖLDY G.

1986 : " Die Truppenkommandeure in den Militärdiplomen ", in E.CK W., WOL.FF H. (EDS), Heer und Integrationspolitik. Die römischen Militärdiplome als historische Quelle, Köln-Wien, Böhlau (coll. Passauer historische Forschungen, 2), p. 384-436.

\section{ASCARI M.}

1942 : La Corsica nell'Antichitì, Istituto per gli studi di politica internazionale, Collana storica corsa, Varesc-Milano, $27 \overline{\mathrm{p}}$.

\section{BLÜMEL W. (ED.)}

1985 : Inschrift. von Iasos, I-Il. Inschriften der Stadt, Bonn, Habelt (coll. Inschriften griechischer Städte aus Kleinasien, 28, 1-2)

1987 : Inschrift. von Mylasa, I. Inschriften der Stadt, Bonn, Habelt (coll. Inschriften griechischer Städte aus Klcinasien, 34).

\section{CAGNat R. (DIR.)}

1906-1927: IGR. Autoritale el Impensis Academiae Inscriptionum et Litterarum Humaniorum Collectae el lidita, I-III, Paris, Lcroux.

\section{CECAMORE C.}

2002 : Palatium. Topografia storica dol Palatino tra III sec. a.C. P I sec. d.C.., Roma, "L'Erma " di Bretschneider (coll. Suppl. au Bullettino della Commissiome archeologica comunale, 9), $264 \mathrm{p}$.

\section{CHAPOT V.}

1896 : La Flotte de Misène, Paris, Leroux.

\section{COLLECTIF}

2004 : Libitina e dintorni. Libitina e I luci sepol-

\section{LiAnnér épigraphique.}

Bibliothèques des Écoles françaises d'Athènes et de Rome.

Corpus Inscriptionum Latinarum.

Inscriptiones Graecae ad Res Romanas Pertinentes.

Inscriptiones Craecae Urbis Romae.

Die Inschriften von Fiphesos.

Die Inschriften von Iasos.

Die Inschriften von Mylasa.

Die Inschriften von Simyrna.

Lexicon Topographicum Vrbis Romae.

Onomasticon Prouinciarum Europae Latinarum.

Prosographia Imperii Romani (2“éd.).

Prosopographia militarium equestrium qui fuerunt ab Augusto ad Gallienum.

Reat-Encyclopädie der classischen Altertumswissenschaft.

Roman Military Diplomas.

Zeitschrift für Papyrologie und Epigraphik.

crali, le leges libitinariae campane, iura sepulchrorum : vecchie e nuove iscrizioni, Actes de la $X I^{\prime}$ rencontre franco-ilalienne sur l'epigraphie du monde romain, 10-11 mai 2002, Rome, Quasar, 652 p.

\section{Creaghan J. S., RaubitscheK A. E.}

1947 : Early Christian Epilaphs from Athens, Woodstock, Woodstock Theological Studies, $54 \mathrm{p}$.

\section{DEGRASSI $\Lambda$.}

1925: "Sul diploma CII del Corpus", Rivista di Filologia e Istruzione Classica, n. s. 3, L.III, p. $527-541$.

1952 : I Fasti consolari dellimpero romano dal 30 avanti Cristo al 613 dopo Cristo, Roma, ed. di Storia e Letteratura (coll. Sussidi cruditi, 3), $287 \mathrm{p}$.

\section{DERDA T.}

1986 : "Eumoiria. A Proper Name or an Epithet of Deceased ", ZPl:, 64, p. 87-90.

\section{DEVIJVER H. (ED.)}

1976-2001 : PME: Symbolae A3, I-VI, Leuven, Universitaire Pers.

\section{DIETZ K.}

2002: " Das Künzinger Militärdiplom und die Fasti Ostienses vom Jahr 160 n. Chr.", (hiron, 32, p. 395-400.

\section{DUŠANIĆ $\mathbf{S}$.}

1984 : "Loci constitutionum fixarum ", Epigraphica, XIVI, 1-2, p. 91-115.

1985: "The Sailor's Calendar. Notes on the Day-Dates of Military Diplomata ", in WEBrer E., DOBFsch G. (EDS), Römische
Geschichte, Altertumskunde und Ephigraphik. Festschrift für Artur Betz zur Vollendung seines 80. Lebensjahres, Wien, Österreich Gesellschaft für Archäologie (coll. Archäologisch-Epigraphische Studien, I), p. 234-248.

\section{ECK W.}

1998 : "Documenti amministrativi : pubblicazione e mezzo di autorappresentazione ", in PACI G. (ED.), Epigrafia romana in area adriatica, Actes de la $I X^{r}$ rencontre francoitalienne sur l'épigraphie du monde romain. Macerala, 10-11 nov. 1995, Pisa-Roma, Istituti editoriali poligrafici internazionali, p. 343-366.

2002 : "Zum Zeitpunkt des Wechsels der tribunicia potestas des Philippus Arabs und andere Kaiser », ZPE, 140, p. 257-261.

2003a : “ Eine Burgerrechtskonstitution Vespasians aus dem Jahr $71 \mathrm{n}$. Chr. und die Aushebung von Brittonischen Auxiliaren ", ZPE, 143, p. 220-233.

2003b: "Eine weitere Kopie der domitianischen Bürgerrechtskonstitution für die Truppen Syriens vom 7. Nov. 88 ", $7 P E$, 143, p. 229-233.

Eck W., Macdonald D., Pangerl A.

2002a : "Neue Militärdiplome für Truppen in Italien : Legio II Adiutrix, Flotten und Prätorianen ", ZPE, 139, p. 195-207.

2002b: "Neue Militärdiplome mit neuen Konsulndaten ", Chiron, 32, p. 401-426. 2002c: "Neuc Diplome für das Heer der Provinz Syria ", Chiron, 32, p. 427-448.

Eck W., Pangerl A.

2003 : «Sex. Iulius Frontinus als Iegat des 
niedergermanischen Heeres. $Z u$ neuen Militärdiplomen in der germanischen Prowinzen ", ZPE, 143, p. 205-219.

\section{ECK W., WEISS P.}

2001 : "Die Sonderregelungen für die Soldatenkinder seit Antoninus Pius. Ein niederpannonisches Militärdiplom vom 11. Aug. 146 », ZPF, 135, p. 195-208.

ECK W., WOLFF H. (EDS)

1986: Heer und Integrationspolitik. Die römischen Militärdiplome als historische Quelle, KölnWien, Böhlau (coll. Passauer historische Forschungen, 2), $615 \mathrm{p}$.

\section{ESPÉRANDIEU E.}

1893: Inscriptions antiques de la Corse, Bastia, Ollagnier.

\section{FORNI G.}

1986 : « I diplomi militari dei classiari delle flotte pretorie (inclusi quelli dei classiarilegionari) ", in E.cK W., WOL.FF H. (EISSi, Heer und Integrationspolitik. Die römischen Militärdiplome als historische Quello, p. 293-321.

FRASER I = FRASER P. M., MATTHEWS E. (EDS)

1987 : A Lexicon of (ireek Personal Names -I- The Aegean Islands, Cyprus, Cyrenaica, Oxford, Clarendon Press, $489 \mathrm{p}$.

FrASER II = FraSer P. M., OSBORNE M. G., BYRNE S. G. (EDS)

1994 : A Iexicon of (ireek Persomal Names -IIAttica, Oxford, Clarendon Press, $510 \mathrm{p}$.

\section{FRASER III = FRASER P. M., MATTHEWS} E. (EDS)

1997 : A Lexicon of (ireek Personal Names-III- The Peloponnese, Western Greece, Sicily and Magna Graecia, Oxford, Clarendon Press, sl9 p.

\section{GIACOMINI P.}

1990 : "Anagrafe dei classiarii ", in SLSINI G. (ED.), Storia di Ravenna, I. L'Evo antico, Ravenna-Venezia, Marsilio, p. 321-362.

\section{GREGORI G. L.}

1990 : Brescia romana. Ricerche di prosopografia e storia sociale, I. I Documenti, Roma, Quasar (coll. Vetera, 7), $299 \mathrm{p}$.

\section{HOLTHEIDE B.}

1983 : Römische Bürgerrechtspolitik, Freiburg, Hochschulverlag (coll. Hochschulsammlung Philosophie Geschichte, 5), $497 \mathrm{p}$.
JEHASSE J., JEHASSE L.

1987 : Aléria antique, Aléria, Les Amis d'Aléria, $117 \mathrm{p}$.

\section{KIENAST D.}

1990 : Römische Kausertabelle. Grundzüge einer römische Kaiserchronologie, Darmstadt, Wissenschaftliche Buchgesellschaft, $377 \mathrm{p}$.

\section{LA ROCCA E.}

2002 : «Premessa (con un appunto sul tempio di Minerva) ", in CECAMORE C., Palatium. Topografa storica del Palatino tra III sec. a.C. e 1 sec. d.c., Roma, "L'Erma " di Bretschneider (coll. Suppl. au Bullettino della (ommissione archeologica comunale, 9), p. 5-8.

LÖRINCZ B. (ED.)

1999 : OPEI, II, Wien, Forschungsgesellschaft Wiener Stadtarchäologie, $232 \mathrm{p}$.

\section{MASTINO A.}

1993: "Tabularium principis e tabularia provinciali nel processo contro i Galillenses della Barbaria sarda ", in La Tavola di Esterzili. Il conflitto tra pastori e contadini nella Barbaria sarda, Convegno di studi, Esterzili, 13.VI.1992, Università degli studi di Sassari, Centro di studi interdisciplinari sulle province romane, Sassari, Gallizzi, p. 99-117.

MERKELBACH R., NOLLÉ J. (EDS)

1980 : Inschrift. von Ephesos, VI. Repertorium nn. 2001-2958, Bonn, Habelt (coll. Inschriften griechischer Städte aus Kleinasien, 16).

1981 : Inschrift. von Ephesos, VII/1. Repertorium nn. 3001-3500, Bonn, Habelt (coll. Inschriften griechischer Städte aus Kleinasien, 17, 1).

\section{Mócsy A.}

1986 : «Die Namen der Diplomempfänger », in ECK W., WOL.FF H. (F.DS), Heer und Integrationspolitik. Die römischen Militärdiplome als historische Quelle, Köln-Wien, Böhlau (coll. Passauer historische Forschungen, 2), p. 437-466.

MORETTI L. (ED.)

1968-1990: IGUR -I-IV- Miscellanea Greca e Romana, 17, Roma, Istituto italiano per la storia antica.

\section{MORRIS C. J., ROXAN M. M.}

1977 : "The Witnesses to Roman Military Diplomata ", Acta Archaeologica Arheoloski Vestnik I jubljana, 28, p. 299-333.
NESSELHAUF H. (ED.)

1936-1955: CII, XVI, Diplomata militaria : indices, Berlin.

PAPI E.

2003 : "Diploma militare da Thamusida (Mauretania Tingitana) : 31 dicembre $133 / 134$ », ZPE, 142, p. 257-266.

PARMA A.

2002 : "Note sull'origine geografica dei classiarii nelle flotte imperiali : i marinai di provenienza nordafricana ", in KHANOUSSI M. LT AL. (EDS), I.Africa romana, XIV. Lo Spazio marittimo del Mediterraneo occidentale, Atti del XIV convegno di studio, Sassari, 7-10.XII.2000, Sassari, Collana del dipartimento di storia dell'Università degli studi di Sassari $\left(2^{\circ}\right.$ séric), Roma, Carocci (coll. Pubblicazioni del Centro di studi interdisciplinari sulle province romane, 13), p. 323-332.

Sous presse : "Onomasticon dei classiarii ", in CAMODECAA G. (ED).), Miseno romana.

PETZl G. (ED.)

1982 : Inschrift. von Simyrna, I. Crabinschriften, postume Ehrungen, Grabefigramme, Bonn, Habelt (coll. Inschriften griechischer Städte aus Klcinasien, 23).

\section{PFLAUM H.-G.}

1961 : Les Carrières procuratoriennes équestres sous le Haut-Empire romain, III, Paris, Paul Geuthner, Institut français d'archéologie de Beyrouth (coll. Bibliothèque archéologique et historique, I.VII), $515 \mathrm{p}$.

\section{REDDÉ M.}

1986: Mare nostrum. Les infrastructures, le dispositif et l'histoire de la marine militaire sous l'tempire romain, Paris-Rome, f́cole française de Rome (coll. BEFAR, 260), $737 \mathrm{p}$.

RosSi A. M.

1975 : " Ricerche sulle multe sepolcrali romane ", Rivista di Storia Antica, 5, p. 111 159 .

\section{ROXAN M. M.}

1978 : $R M D$, I (1954-1977), L.ondon, University of London, Institute of Archaeology, Occasional Publication, 2 [nn. 1-78], $118 \mathrm{p}$.

$1985: R M D$, II (1978-1984), I.ondon, Cniversity of Iondon, Institute of Archacology, Occasional Publication, 9 [nn. 79-135], $231 \mathrm{p}$.

1994 : RWD, III (1985-1993), London, Lniversity College, Lniversity of London, 
Institute of Archacology, Occasional Publication, 14 [nn. 136-201], $362 \mathrm{p}$.

\section{ROXAN M. M., STYLOW A. U.}

1999 : " Ein neues Flottendiplom von 18. Dezember 225 und $R M D$, III, $194=(I I$, $\mathrm{II}^{2} / 7,127 \mathrm{a}$ ", Chiron, 29, p. 182-192.

\section{SADDINGTON D. B.}

1997 : "The Witnessing of Pre- and Early Flavian Military Diplomas and Discharge Procedures in the Roman Army ", Epigraphica, LIX, p. 156-172.

\section{SÁNCHEZ P.}

2001 : " Un diplôme militaire inédit de Sévère Alexandre pour M. Aurclius Bithus (7 janvier 230 apr. J.-C.) ", ZPl, 137, p. $245-250$.

\section{Solin H.}

1982 : Die griechischen Personennamen in Rom, (II. auctarum, I-II. Berlin, De Gruter. 1999 : Die stadtrömischen Sklavennamen. Kin Namenbuch, I-III. Forschungen zum antiken Sklaverei, Stuttgart, Steiner.

\section{STARR C. G.}

1960 : The Roman Imperial Nary, 31 B(-Al) 324, Cambridge, Heffer, 232 p.
STEINBY E. M. (ED.)

1993-2000 : LTUR, I-VI. Roma, Quasar.

\section{STRUBBE J.}

1997 : APAI F Desecrators of the (rrave in the Creek Epitaphs of Asia Minor: a Catalogue, Bomn, Habelt (coll. Inschriften griechischer Städte aus Kleinasien, 52), $368 \mathrm{p}$.

\section{VISMARA C.}

1980 : "Prima miscellanea sulla Corsica romana ", Mélanges de l'École française de Rome, Antiquité, 92, 1, p. 303-328.

1987 : "Funzionari civili e militari nella Corsica romana ", in Studi per Laura Breglia, Suppl. au no 4/1987 (parte III) du Bollettino di Numismatica, p. 57-68.

\section{WEISS $P$.}

1999 : “ Fin Konsulnpaar vom 21. iuni 159 n. Chr. Drei Diplome für Kommilitonen der oberpannonischen Ala I Thracum uirtrix c.R. ", Chiron, 29, p. $147-182$.

2000: " Zu Vicusangaben und qui-et-Namen auf Flottendiplomen des 3. Jh.s ", ZPE, 130, p. $279-285$.
2001 : «Neue ausgewählte Diplomfragmente ", ZPE, 134, p. 267-272.

2002a: "Neue Flottendiplome für Thraker aus Antoninus Pius' späten Jahren », ZPE, 139, p. 219-225.

2002b: "Neue Diplome für Soldaten des Exerritus Dacicus ", ZPE, 141, p. 241-251. 2002c : "Ausgewählte neue Militärdiplome. Seltene Provinzen (Africa, Mauretania (atesariensis), späte Urkunden für Prätorianer (Caracalla, Philippus) ", (.hiron, 32, p. 491-543.

\section{WOLFF H.}

1986 : “Die Entwicklung der Veteranenprivilegien won Beginn des 1. Jahrhunderts v. Chr. Bis auf Konstantin d. Gr. ", in Ec: W., Wol.FF H. (F.DS), Heer und Integrationspolitik. Die römischen Militärdiplome als historische Quelle, Köln-Wien, Böhlau (coll. Passauer historische Forschungen, 2), p. 44-115.

\section{ZUCCA R.}

1996: La Corsica romana, Centro di studi interdisciplinari sulle province romane, Oristano, S'Alvure, 303 p. 